\title{
mumpce_py: A Python Implementation of the Method of Uncertainty Minimization Using Polynomial Chaos Expansions
}

\author{
David A. Sheen \\ National Institute of Standards and Technology, \\ Gaithersburg, MD 20899, USA \\ david.sheen@nist.gov \\ Software DOI: https://doi.org/10.18434/M3Z38C \\ Software Version: 0.1 \\ Key words: experimental database; experimental design; optimization; outlier detection; uncertainty analysis. \\ Accepted: September 28, 2017 \\ Published: October 4, 2017 \\ https://doi.org/10.6028/jres.122.039
}

\section{Summary}

The Method of Uncertainty Minimization using Polynomial Chaos Expansions (MUM-PCE) [1-6] was developed as a software tool to constrain physical models against experimental measurements. These models contain parameters that cannot be easily determined from first principles and so must be measured, and some which cannot even be easily measured. In such cases, the models are validated and tuned against a set of global experiments which may depend on the underlying physical parameters in a complex way. The measurement uncertainty will affect the uncertainty in the parameter values.

MUM-PCE was written to provide a streamlined workflow for computational uncertainty analysis. The software provides the following functionality:

- Compiles a database of experimental measurements

- Compares calculations using a physical model with the measurements (validation)

- Constrains the physical model against the measurements (optimization)

- Determines the model uncertainty based on the measurement uncertainty (uncertainty analysis)

- Identifies measurements inconsistent with the constrained model (outlier detection)

- Identifies experimental measurements that do not strongly constrain the model (experimental design)

The software cannot be used out of the box. Users must create an interface to their own software, and that interface will be specific to the user's application. Two examples of how to write an interface are provided. One is a toy model that is intended to be as complete as possible while also simple. The other is an interface to the reaction kinetics program Cantera (http://cantera.org), which represents a research-level use case with multiple heterogeneous experiments and a highly complex interface to a detailed model. 


\section{Software Specifications}

\begin{tabular}{ll}
\hline NIST Operating Unit(s) & Chemical Sciences Division, Material Measurement Laboratory \\
\hline Category & Optimization and uncertainty analysis \\
\hline Targeted Users & Users of any modeling code wishing to estimate uncertainty \\
\hline Operating System(s) & Cross-platform, where Python, NumPy, and SciPy are installed \\
\hline Programming Language & Python 3.6 \\
\hline Inputs/Outputs & $\begin{array}{l}\text { This software is not usable out-of-the-box. The user must write } \\
\text { additional code to interface their model with this software. }\end{array}$ \\
\hline Documentation & https://pages.nist.gov/mumpce_py \\
\hline Accessibility & N/A small-scale research tool \\
\hline Disclaimer & https://www.nist.gov/director/licensing \\
\hline
\end{tabular}

\section{Methods for Validation}

The software is verified using the toy_model submodule. More details can be found in the documentation at https://pages.nist.gov/toy.html. This model is chosen to have an analytic solution.

\section{References}

[1] Sheen DA, Wang H (2011) The method of uncertainty quantification and minimization using polynomial chaos expansions. Combustion and Flame 158(12):2358-2374. https://doi.org/10.1016/j.combustflame.2011.05.010.

[2] Sheen DA, Wang H (2011) Combustion kinetic modeling using multispecies time histories in shock-tube oxidation of heptane. Combustion and Flame 158(4):645-656. https://doi.org/10.1016/j.combustflame.2010.12.016.

[3] Sheen DA, Rosado-Reyes CM, Tsang W (2013) Kinetics of $\mathrm{H}$ atom attack on unsaturated hydrocarbons using spectral uncertainty propagation and minimization techniques. Proceedings of the Combustion Institute 34(1):527-536. https://doi.org/10.1016/j.proci.2012.06.062.

[4] Sheen DA, Manion JA (2014) Kinetics of the reactions of $\mathrm{H}$ and $\mathrm{CH}_{3}$ radicals with $n$-butane: an experimental design study using reaction network analysis. The Journal of Physical Chemistry A 118(27):4929-4941. https://doi.org/10.1021/jp5041844.

[5] Manion JA, Sheen DA, Awan IA (2015) Evaluated kinetics of the reactions of H and CH3 with n-alkanes: experiments with nbutane and a combustion model reaction network analysis. The Journal of Physical Chemistry A. https://doi.org/10.1021/acs.jpca.5b01004.

[6] Wang H, Sheen DA (2015) Combustion kinetic model uncertainty quantification, propagation and minimization. Progress in Energy and Combustion Science 47(0):1-31. https://doi.org/10.1016/j.pecs.2014.10.002.

About the author: David Sheen is a Physicist at NIST. His work is on uncertainty analysis in complex systems including high-temperature chemical kinetics and metabolomics. The National Institute of Standards and Technology is an agency of the U.S. Department of Commerce. 